\title{
A case of neurofibromatosis 2 presenting with a mononeuritis multiplex
}

\author{
T J Kilpatrick, R J Hjorth, M F Gonzales
}

\begin{abstract}
A patient with neurofibromatosis 2 had an asymmetrical peripheral neuropathy. A nerve biopsy specimen revealed neurofibromatous changes, and the neuropathy may have been a direct consequence of neurofibromatosis. An apparent clinical response to immunosuppressive treatment and plasma exchange is also reported.
\end{abstract}

The term neurofibromatosis defines two genetically and phenotypically distinct disorders. $^{1}$ Neurofibromatosis 1 , or von Recklinghausen's disease, is an autosomal dominant disorder with the genetic abnormality localised to chromosome 17. Typical abnormalities include café-au-lait spots, neurofibromas, and Lisch nodules in the iris. Neurofibromatosis 2 is also an autosomal dominant disorder, but the genetic abnormality is a deletion on chromosome 22 . The characteristic feature is bilateral acoustic neuromas, and loss of hearing is often the first symptom. Other CNS tumours, including meningiomas and gliomas, also occur. Café-au-lait spots and peripheral tumours, although less common than in neurofibromatosis 1, may also be found. ${ }^{2}$ The tumours range from discrete Schwannomas to poorly delineated plexiform neurofibromas. ${ }^{3}$ Even in neurofibromatosis 1, however, peripheral tumours are usually asymptomatic, with deficits occurring in only $4.3 \%$ of cases in the series reported by Cannale et al. ${ }^{4}$

We have found there are five previous reports of peripheral neuropathy associated with neurofibromatosis, and two of the cases had neurofibromatosis $2 .^{5-8} \mathrm{We}$ describe a further patient who had neurofibromatosis 2 , the first to our knowledge to present initially with an asymmetrical sensorimotor neuropathy and subsequently to develop more characteristic features.

\section{Case report}

A 24 year old male presented with four months of progressive peripheral numbness and weakness, beginning with paraesthesiae in the fingers and weakness of the left foot, followed by the development of truncal ataxia and multiple falls. There was bilateral wasting of the periscapular and intrinsic hand muscles and wasting of the left deltoid, brachioradialis, and forearm extensors. There was weakness of the intrinsic muscles of the right hand and generalised weakness of the left arm. The legs were also wasted with symmetrical distal weakness. The reflexes were depressed on the right but normal on the left, except for a depressed ankle jerk. The right plantar was flexor and the left unresponsive. There was a mild impairment of all sensory modalities in the hands and legs, extending to the right knee and to the left anterior superior iliac spine. There were no palpable enlargements along the course of any peripheral nerves.

The results of tests for urea, electrolytes and liver function, full blood examination and random blood glucose and serum calcium, phosphate, vitamin B12, and folate concentrations were all normal. There was no paraproteinaemia, antinuclear antibodies were not detected and the erythrocyte sedimentation rate was $27 \mathrm{~mm}$ in the first hour. Serological tests for syphilis and HIV 1 yielded negative results. The CSF contained $5 \times 10^{\circ} / 1$, the glucose concentration was $3.8 \mathrm{mmol} / \mathrm{l}$, and the protein was elevated at $1.39 \mathrm{~g} / 1$. Nerve conduction studies showed mild slowing of the left ulnar motor conduction velocity to $40 \mathrm{~m} / \mathrm{s}$, and the left median and ulnar and the right lateral popliteal nerve amplitudes were at the lower limits of the normal ranges. The corresponding F wave latencies were also mildly prolonged. The ulnar, median, and left sural nerve sensory potentials were absent, but the right sural nerve action potential was preserved. A left sural nerve biopsy specimen showed loss of some myelinated fibres without active axonal degeneration or demyelination.

A provisional diagnosis of subacute, asymmetrical, sensorimotor peripheral neuropathy was made. Although the nerve conduction studies did not show either a conduction block or gross slowing of the conduction velocity, the clinical presentation and CSF findings suggested the possibility of an inflammatory demyelinating neuropathy, and the patient was treated with prednisolone $(50 \mathrm{mg} /$ day). Two months later, however, there had been progression of symptoms and signs. Azathioprine was started $(100 \mathrm{mg} /$ day $)$, and seven plasma exchanges were given. Considerable clinical improvement occurred over the next three months. In particular, the sensory deficit diminished, his ataxia resolved, and he was able to return to his previous employment.

Two years later he presented with a hoarse voice, right tinnitus, and impaired vision in the 
right eye. There was a right fundal astrocytic hamartoma. Examination also showed right lower motor neuron facial nerve, accessory nerve, and vocal cord pareses and bilateral sensorineural deafness. The pattern of weakness in the limbs was unchanged but in the legs the sensory abnormalities were now confined to the feet. The ankle jerks were absent and both plantar responses were extensor. A cerebral CT scan showed multiple meningiomata and bilateral acoustic neuromas. MRI of the cerebrum and cervical cord confirmed this and also revealed tumours on the second and seventh cervical nerve roots, the former causing cervical cord compression. An MRI of the lower cord and the cauda equina was normal, with no evidence of tumours on the lumbarsacral nerve roots. A diagnosis of neurofibromatosis 2 was made and, on review, small cutaneous neurofibromas were found and confirmed histologically. There was no known relevant family history.

The original sural nerve biopsy specimen was reviewed, and further sectioning detected changes in one fasciculus suggestive of a neurofibroma (figure). As part of the review,
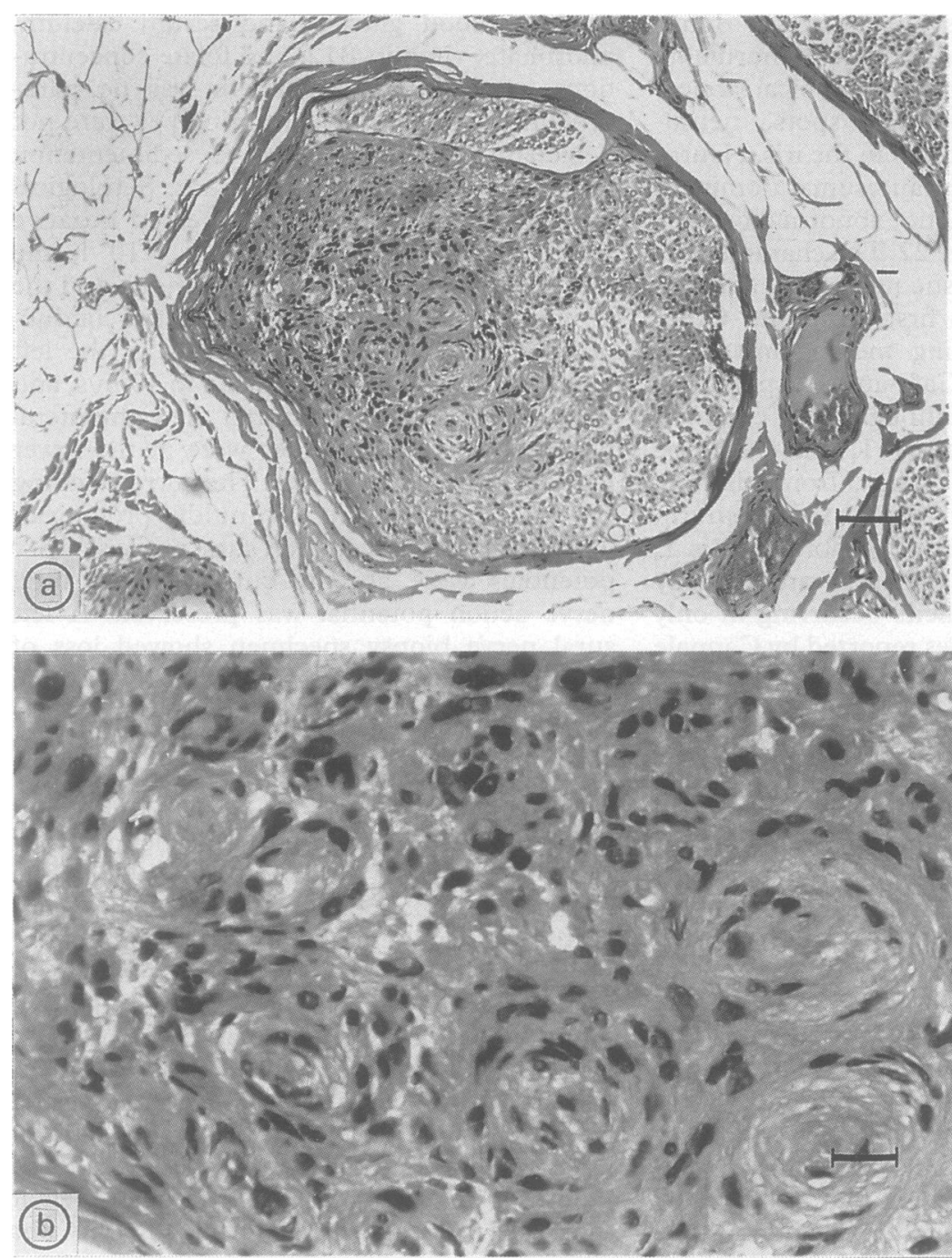

Figure Transverse sections of nerve fasciculus with enlargement showing distortion by whorled structures constituting small endoneurial neurofibroma (haematoxylin and eosin staining) a) (top) magnification bar $=80 \mu \mathrm{m}, b$ ) (bottom) magnification bar $=20 \mu \mathrm{m}$ ).
S-100 staining was performed and immunoreactivity was detected within the whorled structures comprising the endoneurial neurofibroma. The upper cervical tumour was removed surgically and identified as a neurofibroma by histopathology. At the operation, numerous tumours on multiple cervical nerve roots were also detected. The patient made a good recovery after the operation

\section{Discussion}

This patient initially presented with signs suggestive of an asymmetrical peripheral neuropathy, and there was objective improvement after starting immunosuppressive treatment. The subsequent development of cranial nerve palsies led to the identification of multiple central nervous system tumours and to the ultimate diagnosis of neurofibromatosis 2 .

There are five previous reports of generalised neuropathy associated with neurofibromatosis but the clinical features, histological findings, and postulated pathogenetic mechanisms differ. Bradley et $a l^{5}$ reported a family with a predominantly motor axonal neuropathy resembling peroneal muscular atrophy, in which the propositus had neurofibromatosis 1. A sural nerve biopsy specimen revealed multiple tumours, but because one of two daughters with subclinical neuropathy did not have clinical neurofibromatosis, the neurological deficits in the kindred were attributed to an unrelated neuronal degeneration. Bosch et $a l^{6}$ also described four patients, three from one family, with neurofibromatosis 1 and clinical features resembling peroneal muscular atrophy. Nerve biopsy specimens showed axonal degeneration and concentric Schwann cell hyperplasia but lacked classical neurofibromatous changes. The neuropathy was attributed to a primary neuronal degeneration with reactive Schwann cell proliferation.

Ohnishi and $\mathrm{Nada}^{7}$ reported a patient with neurofibromatosis 2 and a six year history of a progressive, distal sensorimotor neuropathy. A nerve biopsy specimen showed large myelinated fibre loss and lamellated structures interpreted as areas of Schwann cell hyperplasia. Thomas et $a,^{8}$ however, reviewed the histology and suggested that these structures were of perineurial origin and characteristic of neurofibromatous changes, making a compressive cause for the neuropathy more likely. They also reported three further patients. The third had neurofibromatosis 2, palpable nerves, and presented with a six month history of a progressive and symmetrical sensorimotor neuropathy. Neurofibromas were identified on a nerve biopsy specimen in this patient, and the authors concluded that the neuropathy was the direct consequence of neurofibromatosis. ${ }^{8}$

Our case differs from the above reports in that an apparent asymmetrical peripheral neuropathy of subacute onset was the initial manifestation. Although individual subcutaneous neurofibromas are often asymptomatic, in this patient the clinical findings suggested that tumours were the direct cause of a 
multiple mononeuropathy. The nerve biopsy specimen finding was also suggestive of a neurofibroma, although the disorganised fascicular pattern and whorl formation were reminiscent of localised hypertrophic neuropathy, ${ }^{9}$ which has also been reported to occur in neurofibromatosis. ${ }^{10}$ If the cause of the peripheral neuropathy was direct compression of the nerve fasciculus by intrinsic tumour, the apparent dramatic response to immunosuppressants and plasma exchange remains unexplained. Delayed inhibition of tumour growth by the antimitotic effects of azathioprine can be postulated but is speculative. There are anecdotal reports suggesting chemotherapy and hormone induced inhibition of Schwannoma growth. Jahrsdoerfer and Benjamin ${ }^{11}$ reported two patients with bilateral acoustic neuromas with progressive hearing loss and tumour growth. After combination chemotherapy there was an apparent stabilisation of hearing, as assessed by audiography and cerebral CT, respectively. Jay et al ${ }^{12}$ reported that in the rat, Schwann cell tumour induction may be inhibited by 17-B estradiol and tamoxifen.
1 Kanter WR, Eldridge R, Fabricant R, Allen JC, Koerber T. Central neurofibromatosis with bilateral acoustic neuromas: genetic, clinical and biochemical distinctions from peripheral neurofibromatosis. Neurology 1980;30:851-9.

2 Martuza RL, Eldridge R. Neurofibromatosis 2. $N$ Engl f Med 1986;318:684-8.

3 Adams JH. The nervous system. In: Anderson JR, ed Muir's textbook of pathology. 10th ed. London: Edward Muir's textbook of pa

4 Cannale D, Bebin J, Knighton RS. Neurologic manifestaCannale $D$, Bebin J, Knighton RS. Neurologic manifesta-
tions of von Recklinghausen's disease of the nervous tions of von Recklinghausen's disease
system. Confin Neurol 1964;24:359-403.

5 Bradley WG, Richardson J, Frew IJC. The familial association of neurofibromatosis, peroneal muscular atrophy, congenital deafness, partial albinism, and Axenfeld's defect. Brain 1974;97:521-32.

6 Bosch EP, Murphy MJ, Cancilla PA. Peripheral neurofibromatosis and peroneal muscular atrophy. Neurology 1981;31:1408-14.

7 Ohnishi $\mathrm{A}, \mathrm{Nada} \mathrm{O}$. Ultrastructure of the onion bulb-like structure observed in the sural nerve in a case of von Recklinghausen's disease. Acta Neuropathol (Berl) 1972; 20:258-63.

8 Thomas PK, King RHM, Chiang TR, Scarravili F, Sharma AK, Downie AW. Neurofibromatous neuropathy. Muscle Nerve 1990;13:93-101.

9 Bilbao JM, Khoury NJS, Hudson AR, Briggs SR. Perineurioma (localised hypertrophic neuropathy). Arch Pathol Lab Med 1984; 108:557-60.

10 Mitsumoto H, Wilbourn AJ, Goren H. Perineurioma as the cause of localized hypertrophic neuropathy. Muscle Nerve cause of localized

11 Jahrsdoerfer RA, Benjamin RS. Chemotherapy of bilateral acoustic neuromas. Otolaryngology-Head Neck Surg 1988; 98:273-82.

12 Jay JR, MacLaughlin DT, Badger TM, Miller DC, Martuza $\mathrm{RL}$. Hormonal modulation of Schwann cell tumors. Annals NY Acad Sci 1986;486:371-82. 